G. Health hazards of smoking and alcohol use

$\mathrm{H}$. Harms of discontinuing treatment when they felt well

Results: A total of 450 patients included spondyloarthropathy $150(34 \%)$, rheumatoid arthritis $200(45.7 \%)$, psoriatic arthritis $45(10 \%)$, and others $25(5.5 \%)$. The following observations was made:

1.Self-reported adherence to medication was in 250 (55\%) patients; 200(45\%) patient were non-adherent to treatment

$2.382 / 450(85 \%)$ patients had misbelieves regarding different food items.

$3.225 / 450(50) \%$ of the patients were not doing regular physiotherapy they were totally dependent on medications for symptoms relief.

$4.387 / 450(86 \%)$ patients confused the symptoms of osteoarthritis with that of RA.

$5.315 / 450(70 \%)$ patients did not feel the requirement of continuing drugs during remission.

$6.135 / 450(30 \%)$ patients believed that while on DMARDs they cannot contemplate pregnancy.

$7.351 / 450(78 \%)$ patients accept the need for vaccination when staring DMARDs $8.360 / 450$ (80\%) patients aware about side effect of smoking in disease but only $40 \%$ were able to quit.

$9.273 / 450(60 \%)$ patients felt that more expensive medicines e.g.bDMARDs have more effects.

$10.360 / 450(80 \%)$ patients believed that DMARDs were 'steroids' and they increased weight. On analysis one patient have more than two myths simultaneously.

Conclusion: Increased awareness of the patient's beliefs about medicines is needed among health care providers. We should encourage patients to express their views about medicines as well as disease in order to optimize and personalize the information process. This can stimulate concordance and adherence to medication and follow up. These myths are deeply rooted in our society, single sitting counseling is not enough, and reinforcement is needed.

References:

[1] Tom Greenhalgh. Facts about rheumatoid arthritis: 7 myths you may encounter. Rheumatology Advisor. March 28, 2019.

Acknowledgments: no

Disclosure of Interests: None declared

DOI: 10.1136/annrheumdis-2020-eular.3099

\section{SAT0646-HPR PATTERN AND INFLUENTIAL FACTORS IN PROMOTING TREAT-TO-TARGET (T2T) FOR FOLLOW-UP OF ANKYLOSING SPONDYLITIS (AS) PATIENTS WITH A RHEUMATOLOGIST-PATIENT INTERACTIVE SMART SYSTEM OF DISEASE MANAGEMENT (SSDM): A COHORT STUDY FROM CHINA}

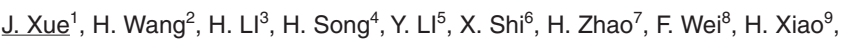
B. Wu ${ }^{9}$, Y. Jia ${ }^{9}, \mathrm{~F} \mathrm{Xiao}^{9}, \mathrm{H}$. Wu $\mathrm{Wu}^{1}$ on behalf of SSDM Collaboration Group, China. ${ }^{1}$ The Second Affiliated Hospital of Zhejiang University school of Medicine, Hangzhou, China; ${ }^{2}$ The First Hospital of Jiaxing, Jiaxing, China; ${ }^{3}$ The Affiliated Hospital of Inner Mongolia Medical University, Neimenggu, China; ${ }^{4}$ Beijing Jishuitan Hospital, Beijing, China; ${ }^{5}$ Zhejiang Provincial People's Hospital, Hangzhou, China; ${ }^{6}$ The First Affiliated Hospital of Henan University of science and technology, Luoyang, China; ${ }^{7}$ Xiangya Hospital of Central South University, Changsha, China; ${ }^{8}$ Jiangmen Central Hospital Affiliated Jiangmen Hospital of Sun Yat-Sen University, Jiangmen, China; ${ }^{9}$ Shanghai Gothic Internet Technology Co., Ltd., Shanghai, China

Background: Ankylosing Spondylitis Disease Activity Score (ASDAS) is adopted to evaluate the degree of disease activity and the inflammatory response in AS patients. ASDAS score <= 1.3 represents inactive disease status and achievement of T2T. SSDM is a mobile application for disease management.

Objectives: To evaluate the patterns of T2T and related influential factors among AS patients after applying SSDM in the real world.

Methods: AS Patients were trained to master SSDM by healthcare professionals (HCPs) and to conduct ASDAS self-assessments. Patients were also required for repeating self-assessments after leaving the hospital. After entry by patients, data can be synchronized to the SSDM terminal of authorized rheumatologists. Based on these data, the patients can apply for consultation to their physicians and rheumatologists can provide medical advices to their patients.

Results: From Jan 2015 to Jan 2020, 17,870 AS patients enrolled in SSDM with the mean age of $34.62 \pm 10.98$ years old and the median disease duration of 3.58 years. Among them, 1,127 AS patients from 150 hospitals were followed up for more than 6 months through SSDM. The results at baseline and in final follow up were summarized in Table 1.
Table 1. The T2T results at baseline and in final follow up.

\begin{tabular}{lcccccccccc}
\hline $\begin{array}{l}\text { BaselinelFinal } \\
\text { follow-up }\end{array}$ & $n$ & $\%$ & $\begin{array}{c}\mathrm{x}<= \\
1.3\end{array}$ & $\begin{array}{l}1.3<\mathrm{x} \\
<=2.1\end{array}$ & $\begin{array}{l}2.1<\mathrm{x} \\
<=3.5\end{array}$ & $\begin{array}{l}3.5 \\
<\mathrm{x}\end{array}$ & $\%$ \\
\hline $\mathrm{x}<=1.3$ & 315 & $27.95 \%$ & 206 & $65.40 \%$ & 74 & $23.49 \%$ & 26 & $8.25 \%$ & 9 & $2.86 \%$ \\
$1.3<\mathrm{x}<=2.1$ & 340 & $30.17 \%$ & 138 & $40.59 \%$ & 114 & $33.53 \%$ & 75 & $22.06 \%$ & 13 & $3.82 \%$ \\
$2.1<\mathrm{x}<=3.5$ & 363 & $32.21 \%$ & 95 & $26.17 \%$ & 106 & $29.20 \%$ & 133 & $36.64 \%$ & 29 & $7.99 \%$ \\
$3.5<\mathrm{x}$ & 109 & $9.67 \%$ & 24 & $22.02 \%$ & 25 & $22.94 \%$ & 42 & $38.53 \%$ & 18 & $16.51 \%$ \\
Total & 1,127 & $100 \%$ & 463 & $41.08 \%$ & 319 & $28.31 \%$ & 276 & $24.49 \%$ & 69 & $6.12 \%$ \\
\hline
\end{tabular}

The rate of T2T achievers were $27.95 \%(315 / 1,127)$ at baseline, and improved significantly to $41.08 \%(463 / 1,127)$ after 6 months follow up, $p<0.01$. Among T2T achievers at baseline, 65.40\% (206/315) maintained T2T, 34.60\% (109/315) relapsed. Of patients who didn't achieve T2T at baseline, only $31.65 \%(257 / 812)$ achieved T2T after 6 months follow up.

The impact of the online interaction between patients and physicians and the frequency of self-assessment for ASDAS on T2T has been analyzed. Compared with 544 patients who didn't interact online with their physicians and self-assessed less than 3times, 104 patients with online interaction and monthly assessments achieved significant higher improvement rate of T2T $(9.19 \%$ vs $23.08 \%$, $\mathrm{p}<0.01$ ). The more frequent of the self-assessments being performed by patients, the higher improvement of T2T rate will be. The improvement of T2T rate(y) was positively correlated with times of self-assessment for ASDAS(x) independently. The regression equation as " $y=0.0304 x+0.0521$ ", $r=0.9107, p<0.01$ (Figure 1).

Figur1. The correlation between the improvement of T2T rate(y) and the times of self-assessment for ASDAS $(x)$

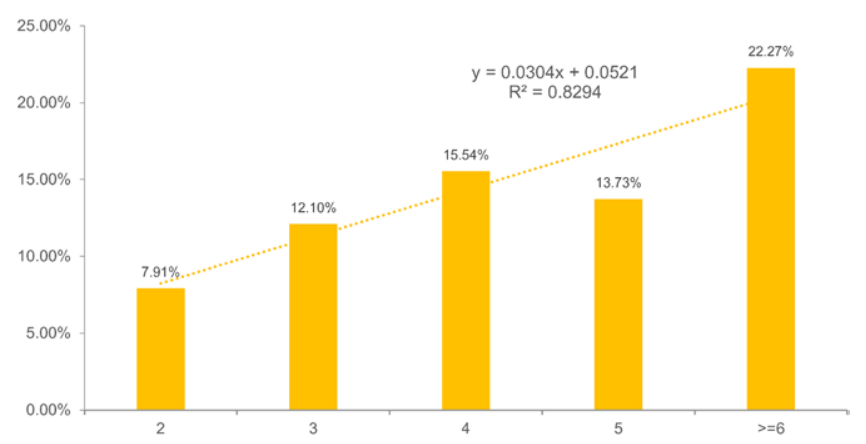

Figure 1.

Conclusion: Significant improvement was observed under applying SSDM through empowering AS patients. After proactive disease management via SSDM for more than 6 months, patients with ASDAS $<=1.3$ score at baseline had a significantly higher retention rate of inactive disease activity. The patients who performed more frequent self-assessments had lower probability of relapse and higher rate of T2T. Online interaction between patients and physicians contributed to promote the improvement rate of T2T. SSDM is a valuable tool for long term follow-up through empowering patients.

Acknowledgments: SSDM was developed by Shanghai Gothic Internet Technology Co., Ltd.

Disclosure of Interests: None declared

DOI: 10.1136/annrheumdis-2020-eular.1798

\section{SAT0647-HPR DEVELOP A MACHINE LEARNING MODEL AND ALGORITHM BASED ON SMART SYSTEM OF DISEASE MANAGEMENT (SSDM) BIG DATA FOR RA FLARE PREDICTION}

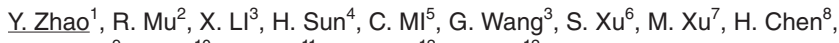
Q. Huang ${ }^{9}$, L. Lei ${ }^{10}$, S. Haili $i^{11}, X$. Chen ${ }^{12}$, F. Xiao ${ }^{12}$ on behalf of SSDM Collaboration Group, China. ${ }^{1}$ Peking Union Medical College Hospital, Beijing, China; ${ }^{2}$ People's Hospital, Beijing University Medical School, Beijing, China; ${ }^{3}$ the First Affiliated Hospital of University of Science and Technology of China, Hefei, China; ${ }^{4}$ Shandong Provincial Hospital Affiliated to Shandong University, Jinan, China; ${ }^{5}$ The Second Affiliated Hospital of Guangxi Medical University, Nanning, China; ${ }^{6}$ the First Affiliated Hospital of Anhui Medical University, Hefei, China; ${ }^{7}$ Affiliated Hospital of Hebei University, Baoding, China; ${ }^{8}$ The Third Affiliated Hospital of Hebei Medical University, Shijiazhuang, China; ${ }^{9}$ Guangdong Provincial Hospital of Chinese Medicine, Guangzhou, China;

${ }^{10}$ The First Affiliated Hospital of Guangxi Medical University, Nanning, China;

${ }^{11}$ Second Hospital of Lanzhou University, Lanzhou, China; ${ }^{12}$ Shanghai Gothic Internet Technology Co., Ltd., Shanghai, China 
Background: Flare, relapse from status of treat-to-target (T2T, DAS28<=3.2), is hard predicted. We try to make it predictable by applying machine learning to a database from smart system of disease management (SSDM). SSDM is an interactive mobile disease management APPs.

Objectives: To develop and validate machine learning algorithms for flare prediction in RA.

Methods: Patients were trained using SSDM and input their data, including demographic, comorbidities (COMBs), lab test, medications and monthly self-assessments, including DAS28, HAQ, SF-36, Hospital Anxiety and Depression Scale (HADS). The data was uploaded to cloud and synchronized to the mobile of authorized rheumatologists. The COMBs were by ICD-9, and medications were listed as cDMARDs, Bio (BioDMARDs), NSAIDs, Steroid, FS (food supplements), MC (medicine for COMBs), TCM (Traditional Chinese Medicine), and combinations.

Results: From Jan of 2015 to Jan of 2020, 8811 RA patients, $85 \%$ female and $15 \%$ male, used to reach T2T. 4556 were flare-free and 4255 suffering at least one flare. The average 160 attributes were extracted from each flare-free patient at time of reaching T2T, and each flare patients at time of 3 months before the flare. Patients were randomly assigned as model setup (training) group (70\%) and validation (testing) group30\%.

For training, data were processed using Python with statistical analyses in $R$. In $R$, random forests were implemented. Logistic regression via glm in base $\mathrm{R}$. The random forest comprises a set of decision trees. "Splits" in the decision trees reflect binary (i.e., yes/no) respect to attributors. Bootstrapping was used to assess, quantify, and adjust for model optimism. Model performance was evaluated using $A \cup C$, precision and recall metrics. Brier scores for accuracy of probabilistic predictions ranged from 0 to 1 ( 0 is perfect discrimination).

The testing showed model performance for prediction windows are 0.78 for AUC (95\% Cl), 0.71 for Recall (sensitivity), 0.195 for Brier score, and 0.68 for precision (true positive 893 , false positive 417 , false negative 367 , true negative 966).

Based on weighing in the random forest, the top 10 pro-flare attributes were CRP, swollen joint count (SJC), tender joint count (TJC), HAQ, DAS28, morning stiffness, gout, MCTD, OA, duration; while top 10 anti-flare attributes were cDMARDs+Bio, cDMARDs+steroid+NSAIDs, stable on HAQ, on morning stiffness, on SJC, medicine on COMBs, cDMARDs+TCM, stable on TJC, on ESR, income at 100-200k (Fig.1). The top weighing COMBs for pro-flaring were gout (0.81), MRD (0.75), OA (0.56), AS (0.48). The monotherapies with either Bio or NSAIDs, or steroid, or TCM was pro-flare; while with cDMARDs was anti-flare $(-0.21)$.

Figure1. Coefficient of features influencing RA relapse

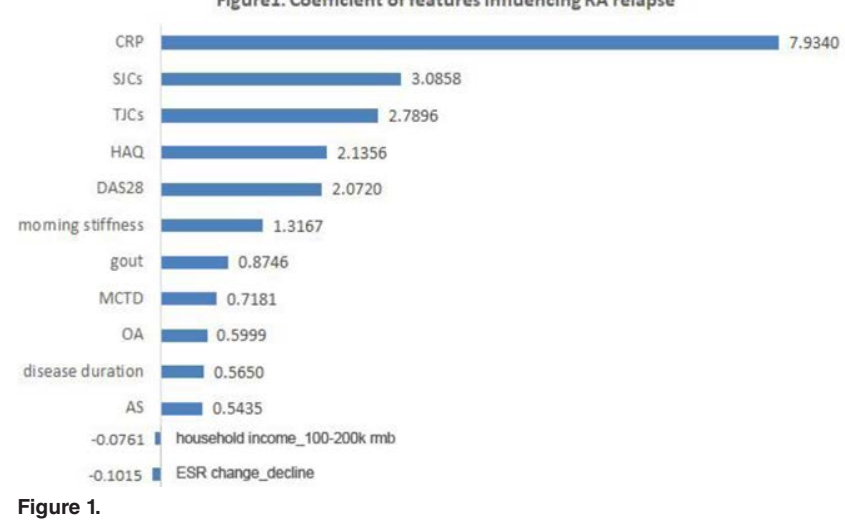

Conclusion: The attempt to develop a machine learning algorithm for RA flare prediction is successful. The discrimination was acceptable. The attributes of both pro-flare and anti-flare are identified, which may inspire the proactive intervention.

Acknowledgments: SSDM was developed by Shanghai Gothic Internet Technology Co., Ltd.

Disclosure of Interests: None declared

DOI: 10.1136/annrheumdis-2020-eular.5458

\section{SATURDAY, 06 JUNE 2020}

\section{HPR Service developments, innovation and eco- nomics in healthcare}

\section{SAT0648-HPR CAN A SPECIALIST NURSE REPLACE A CONSULTANT - AN INNOVATIVE MODEL FOR OSTEOPOROSIS CARE}

J. Begum ${ }^{1}$, J. Fourmy ${ }^{1}$, M. K. Nisar ${ }^{1} .{ }^{1}$ Luton and Dunstable University Hospital, Luton, United Kingdom
Background: Specialist services are heavily reliant on a consultant reviewing a patient and discussing management options. However this can significantly delay treatment pathway owing to lack of sufficient consultant appointments. Clinical nurse specialists (CNSs) are an integral part of a multidisciplinary team employed to provide effective care for the diverse needs of patients with chronic conditions such as osteoporosis.

Objectives: We designed an innovative proof-of-concept osteoporosis service with patients only consulting a metabolic bone CNS and a consultant providing remote oversight. The aim of the project was to improve the efficiency of the service by eliminating consultant appointments and reducing unnecessary hospital visits whilst continuing to deliver a high-quality and safe service.

Methods: A new pathway was implemented where a consultant rheumatologist and a CNS virtually triaged post menopausal women over the age of 65 into the service. A dedicated proforma provided the template for the CNS to undertake new patient telephone consultation. Relevant investigations were requested during the telephone clinic and treatment related information was despatched to help with shared decision making. All patients were then reviewed in a consultant-CNS virtual MDT. Appropriate parenteral treatment option was agreed and confirmed to each individual. The CNS worked through a safety checklist and provided further advice and support to the patient as necessary. Using the database, we compared the timelines for patient journey to conventional pathway, obtained the number of consultant follow-up appointments saved by implementing this service and calculated total savings.

Results: In the proof-of-concept phase, 60 patients were triaged into the new service. It was a combination of 25 new referrals and 35 patients pulled from the consultants' waiting list. Mean age of participants was 77.2 years (65-92) Referral to virtual triage took median 20 days (0-62). Median time for triage to new patient CNS telephone consultation was 18 days (6-87). Time to virtual MDT for treatment authorisation was median zero days (0-76 days). 19 patients had anabolic therapy commenced via home care. Remaining had anti resorptive therapy. No patient requested face-to-face review. Only one patient fed back that they would've preferred to see the consultant once. Sixty new patient consultant appointments were saved and median delay in treatment commencement was reduced from 84 to 38 days.

Conclusion: To our knowledge, this is the first successful example of an innovative service wholly provided by CNSs for commencing parenteral anti-osteoporotic therapy with only remote consultant supervision. Our service redesign has significantly improved the efficiency of the parenteral osteoporosis pathway with reduction in treatment delay and a more streamlined patient journey. A nurse-delivered osteoporosis treatment pathway is highly effective, safe and provides an innovative solution to thinly stretched health care needs of people with chronic conditions.

Disclosure of Interests: Julie Begum: None declared, Joanne Fourmy: None declared, Muhammad Khurram Nisar Grant/research support from: Muhammad Nisar undertakes clinical trials and received support (including attendance at conferences, speaker fees and honoraria) from Roche, Chugai, MSD, Abbvie Pfizer, BMS, Celgene, Novartis and UCB

Consultant of: Muhammad Nisar undertakes clinical trials and received support (including attendance at conferences, speaker fees and honoraria) from Roche, Chugai, MSD, Abbvie, Pfizer, BMS, Celgene, Novartis and UCB

, Speakers bureau: Muhammad Nisar undertakes clinical trials and received support (including attendance at conferences, speaker fees and honoraria) from Roche, Chugai, MSD, Abbvie, Pfizer, BMS, Celgene, Novartis and UCB DOI: 10.1136/annrheumdis-2020-eular.1986

\section{SAT0649-HPR DIRECT COSTS OF SYSTEMIC LUPUS ERYTHEMATOSUS IN COLOMBIA}

J. S. Castro Villarreal ${ }^{1}$, A. Beltrán-Ostos ${ }^{2}$, C. F. Valencia Arboleda ${ }^{1} .{ }^{1}$ University of Los Andes, Industrial Engineering Department, Bogotá, Colombia; ${ }^{2}$ Central Military Hospital, Scientific Research Unit, Bogotá, Colombia

Background: Treatment burden of Systemic Lupus Erythematosus (SLE) is considered high. There are no studies in Colombia that includes the estimation of an incremental cost associated to SLE.

Objectives: To estimate the direct cost associated to SLE in contributory healthcare scheme in Colombia. To estimate prevalence and characterize SLE population affiliated in the contributory healthcare scheme in Colombia. To estimate the direct healthcare cost in patients with and without SLE and the effect of being diagnosed with SLE in the total direct cost during a period of two years. 